Commun. Korean Math. Soc. 27 (2012), No. 3, pp. 523-535

http://dx.doi.org/10.4134/CKMS.2012.27.3.523

\title{
FUZZY STABILITY OF THE CAUCHY ADDITIVE AND QUADRATIC TYPE FUNCTIONAL EQUATION
}

\author{
SunSoOK Jin AND YANG-Hi LeE
}

\begin{abstract}
In this paper, we investigate a fuzzy version of stability for the functional equation

$2 f(x+y)+f(x-y)+f(y-x)-3 f(x)-f(-x)-3 f(y)-f(-y)=0$

in the sense of M. Mirmostafaee and M. S. Moslehian.
\end{abstract}

\section{Introduction}

A classical question in the theory of functional equations is "when is it true that a mapping, which approximately satisfies a functional equation, must be somehow close to an exact solution of the equation?". Such a problem, called $a$ stability problem of the functional equation, was formulated by S. M. Ulam [22] in 1940. In the next year, D. H. Hyers [6] gave a partial solution of Ulam's problem for the case of approximate additive mappings. Subsequently, his result was generalized by T. Aoki [1] for additive mappings, and by Th. M. Rassias [20] for linear mappings, to considering the stability problem with unbounded Cauchy differences. During the last decades, the stability problems of functional equations have been extensively investigated by a number of mathematicians, see [4], [5], [7], [9], [10], [12]-[16], [21].

In 1984, A. K. Katsaras [8] defined a fuzzy norm on a linear space to construct a fuzzy structure on the space. Since then, some mathematicians have introduced several types of fuzzy norm in different points of view. In particular, T. Bag and S. K. Samanta [2], following Cheng and Mordeson [3], gave an idea of a fuzzy norm in such a manner that the corresponding fuzzy metric is of Kramosil and Michalek type [11]. In 2008, A. K. Mirmostafaee and M. S. Moslehian [18] obtained a fuzzy version of stability for the Cauchy functional equation:

$$
f(x+y)-f(x)-f(y)=0 .
$$

Received March 14, 2011

2010 Mathematics Subject Classification. Primary 39B52.

Key words and phrases. fuzzy normed space, fuzzy almost quadratic-additive mapping, Cauchy additive and quadratic type functional equation. 
In the same year, they [17] proved a fuzzy version of stability for the quadratic functional equation:

$$
f(x+y)+f(x-y)-2 f(x)-2 f(y)=0 .
$$

A solution of (1.1) is called an additive mapping and a solution of (1.2) is called a quadratic mapping. Now we consider the functional equation:

(1.3) $2 f(x+y)+f(x-y)+f(y-x)-3 f(x)-f(-x)-3 f(y)-f(-y)=0$

which is called a Cauchy additive and quadratic type functional equation. A solution of (1.3) is called a quadratic-additive mapping. In 2008, C.-G. Park [19] obtained a stability of the functional equation (1.3) by taking and composing an additive mapping $A$ and a quadratic mapping $Q$ to prove the existence of a quadratic-additive mapping $F$ which is close to the given mapping $f$. In his processing, $A$ is approximate to the odd part $\frac{f(x)-f(-x)}{2}$ of $f$ and $Q$ is close to the even part $\frac{f(x)+f(-x)}{2}$ of it, respectively.

In this paper, we get a general stability result of the Cauchy additive and quadratic type functional equation (1.3) in the fuzzy normed linear space in the manner of A. K. Mirmostafaee and M. S. Moslehian [17]. To do it, we introduce a Cauchy sequence $\left\{J_{n} f(x)\right\}$ starting from a given mapping $f$, which converges to the desired mapping $F$ in the fuzzy sense. As we mentioned before, in previous studies of stability problem of (1.3), he attempted to get stability theorems by handling the odd and even part of $f$, respectively. According to our proposal in this paper, we can take the desired approximate solution $F$ at only one time. Therefore, this idea is a refinement with respect to the simplicity of the proof.

\section{Main results}

We use the definition of a fuzzy normed space given in [2] to exhibit a reasonable fuzzy version of stability for the quadratic-additive type functional equation in the fuzzy normed linear space.

Definition $2.1([2])$. Let $X$ be a real linear space. A function $N: X \times \mathbb{R} \rightarrow$ $[0,1]$ (the so-called fuzzy subset) is said to be a fuzzy norm on $X$ if for all $x, y \in X$ and all $s, t \in \mathbb{R}$,

(N1) $N(x, c)=0$ for $c \leq 0$;

(N2) $x=0$ if and only if $N(x, c)=1$ for all $c>0$;

(N3) $N(c x, t)=N(x, t /|c|)$ if $c \neq 0$;

(N4) $N(x+y, s+t) \geq \min \{N(x, s), N(y, t)\}$;

(N5) $N(x, \cdot)$ is a non-decreasing function on $\mathbb{R}$ and $\lim _{t \rightarrow \infty} N(x, t)=1$.

The pair $(X, N)$ is called a fuzzy normed linear space. Let $(X, N)$ be a fuzzy normed linear space. Let $\left\{x_{n}\right\}$ be a sequence in $X$. Then $\left\{x_{n}\right\}$ is said to be convergent if there exists $x \in X$ such that $\lim _{n \rightarrow \infty} N\left(x_{n}-x, t\right)=1$ for all $t>0$. In this case, $x$ is called the limit of the sequence $\left\{x_{n}\right\}$ and we denote it by $N-\lim _{n \rightarrow \infty} x_{n}=x$. A sequence $\left\{x_{n}\right\}$ in $X$ is called Cauchy if for each 
$\varepsilon>0$ and each $t>0$ there exists $n_{0}$ such that for all $n \geq n_{0}$ and all $p>0$ we have $N\left(x_{n+p}-x_{n}, t\right)>1-\varepsilon$. It is known that every convergent sequence in a fuzzy normed space is Cauchy. If each Cauchy sequence is convergent, then the fuzzy norm is said to be complete and the fuzzy normed space is called $a$ fuzzy Banach space.

Let $(X, N)$ be a fuzzy normed space and $\left(Y, N^{\prime}\right)$ a fuzzy Banach space. For a given mapping $f: X \rightarrow Y$, we use the abbreviation

$D f(x, y):=2 f(x+y)+f(x-y)+f(y-x)-3 f(x)-f(-x)-3 f(y)-f(-y)$ for all $x, y \in X$. For given $q>0$, the mapping $f$ is called a fuzzy q-almost quadratic-additive mapping, if

$$
N^{\prime}(D f(x, y), t+s) \geq \min \left\{N\left(x, s^{q}\right), N\left(y, t^{q}\right)\right\}
$$

for all $x, y \in X$ and all $s, t \in(0, \infty)$. Now we get the general stability result in the fuzzy normed linear space.

Theorem 2.2. Let $q$ be a positive real number with $q \neq \frac{1}{2}, 1$. And let $f$ be $a$ fuzzy q-almost quadratic-additive mapping from a fuzzy normed space $(X, N)$ into a fuzzy Banach space $\left(Y, N^{\prime}\right)$. Then there is a unique quadratic-additive mapping $F: X \rightarrow Y$ such that

$$
N^{\prime}(F(x)-f(x), t) \geq \begin{cases}\sup _{t^{\prime}<t} N\left(x,\left(2-2^{p}\right)^{q} t^{\prime q}\right) & \text { if } q>1, \\ \sup _{t^{\prime}<t} N\left(x,\left(\frac{\left(4-2^{p}\right)\left(2^{p}-2\right)}{2}\right)^{q} t^{\prime q}\right) & \text { if } \frac{1}{2}<q<1 \\ \sup _{t^{\prime}<t} N\left(x,\left(2^{p}-4\right)^{q} t^{\prime q}\right) & \text { if } 0<q<\frac{1}{2}\end{cases}
$$

for each $x \in X$ and $t>0$, where $p=1 / q$.

Proof. It follows from $(2.1)$ and $(\mathrm{N} 4)$ that

$$
N^{\prime}(f(0), t)=N^{\prime}(D f(0,0), 4 t) \geq N\left(0,(2 t)^{q}\right)=1
$$

for all $t \in(0, \infty)$. By $(\mathrm{N} 2)$, we have $f(0)=0$. We will prove the theorem in three cases, $q>1, \frac{1}{2}<q<1$, and $0<q<\frac{1}{2}$.

Case 1. Let $q>1$ and let $J_{n} f: X \rightarrow Y$ be a mapping defined by

$$
J_{n} f(x)=\frac{1}{2}\left(4^{-n}\left(f\left(2^{n} x\right)+f\left(-2^{n} x\right)\right)+2^{-n}\left(f\left(2^{n} x\right)-f\left(-2^{n} x\right)\right)\right)
$$

for all $x \in X$. Notice that $J_{0} f(x)=f(x)$ and

(2.3) $J_{j} f(x)-J_{j+1} f(x)=\frac{2^{j+1}-1}{4^{j+2}} D f\left(-2^{j} x,-2^{j} x\right)-\frac{2^{j+1}+1}{4^{j+2}} D f\left(2^{j} x, 2^{j} x\right)$

for all $x \in X$ and $j \geq 0$. Together with (N3), (N4) and (2.1), this equation implies that if $n+m>m \geq 0$, then

$$
N^{\prime}\left(J_{m} f(x)-J_{n+m} f(x), \sum_{j=m}^{n+m-1} \frac{1}{2}\left(\frac{2^{p}}{2}\right)^{j} t^{p}\right)
$$




$$
\begin{aligned}
& \geq N^{\prime}\left(\sum_{j=m}^{n+m-1}\left(J_{j} f(x)-J_{j+1} f(x)\right), \sum_{j=m}^{n+m-1} \frac{1}{2}\left(\frac{2^{p}}{2}\right)^{j} t^{p}\right) \\
& \geq \min \bigcup_{j=m}^{n+m-1}\left\{N^{\prime}\left(J_{j} f(x)-J_{j+1} f(x), \frac{1}{2}\left(\frac{2^{p}}{2}\right)^{j}\right) t^{p}\right\} \\
& \geq \min \bigcup_{j=m}^{n+m-1}\left\{\operatorname { m i n } \left\{N^{\prime}\left(-\frac{\left(2^{j+1}+1\right) D f\left(2^{j} x, 2^{j} x\right)}{4^{j+2}}, \frac{\left(2^{j+1}+1\right) 2^{j p} t^{p}}{8 \cdot 4^{j}}\right),\right.\right. \\
& \geq \min \bigcup_{j=m}^{n+m-1}\left\{N\left(2^{j} x, 2^{j} t\right)\right\}=N(x, t)
\end{aligned}
$$

for all $x \in X$ and $t>0$. Let $\varepsilon>0$ be given. Since $\lim _{t \rightarrow \infty} N(x, t)=1$, there is $t_{0}>0$ such that

$$
N\left(x, t_{0}\right) \geq 1-\varepsilon
$$

Observe that for some $\tilde{t}>t_{0}$, the series $\sum_{j=0}^{\infty} \frac{1}{2}\left(\frac{2^{p}}{2}\right)^{j} \tilde{t}^{p}$ converges for $p=\frac{1}{q}<$ 1. It guarantees that, for an arbitrary given $c>0$, there exists $n_{0} \geq 0$ such that

$$
\sum_{j=m}^{n+m-1} \frac{1}{2}\left(\frac{2^{p}}{2}\right)^{j} \tilde{t}^{p}<c
$$

for each $m \geq n_{0}$ and $n>0$. Together with (N5) and (2.4), this implies that

$$
\begin{aligned}
& N^{\prime}\left(J_{m} f(x)-J_{n+m} f(x), c\right) \\
\geq & N^{\prime}\left(J_{m} f(x)-J_{n+m} f(x), \sum_{j=m}^{n+m-1} \frac{1}{2}\left(\frac{2^{p}}{2}\right)^{j} \tilde{t}^{p}\right) \\
\geq & N(x, \tilde{t}) \geq N\left(x, t_{0}\right) \geq 1-\varepsilon
\end{aligned}
$$

for all $x \in X$. Hence $\left\{J_{n} f(x)\right\}$ is a Cauchy sequence in the fuzzy Banach space $\left(Y, N^{\prime}\right)$, and so we can define a mapping $F: X \rightarrow Y$ by

$$
F(x):=N^{\prime}-\lim _{n \rightarrow \infty} J_{n} f(x) .
$$

Moreover, if we put $m=0$ in (2.4), we have

$$
N^{\prime}\left(f(x)-J_{n} f(x), t\right) \geq N\left(x, \frac{t^{q}}{\left(\sum_{j=0}^{n-1} \frac{1}{2}\left(\frac{2^{p}}{2}\right)^{j}\right)^{q}}\right)
$$


for all $x \in X$. Next we will show that $F$ is the desired quadratic additive function. Using (N4), we have

$$
\begin{aligned}
& N^{\prime}(D F(x, y), t) \\
\geq \min \left\{N^{\prime}\left(2 F(x+y)-2 J_{n} f(x+y), \frac{t}{14}\right), N^{\prime}\left(F(x-y)-J_{n} f(x-y), \frac{t}{14}\right),\right. & N^{\prime}\left(F(y-x)-J_{n} f(y-x), \frac{t}{14}\right), N^{\prime}\left(3 J_{n} f(x)-3 F(x), \frac{t}{14}\right), \\
& N^{\prime}\left(J_{n} f(-x)-F(-x), \frac{t}{14}\right), N^{\prime}\left(3 J_{n} f(y)-3 F(y), \frac{t}{14}\right), \\
& \left.N^{\prime}\left(J_{n} f(-y)-F(-y), \frac{t}{14}\right), N^{\prime}\left(D J_{n} f(x, y), \frac{t}{2}\right)\right\}
\end{aligned}
$$

for all $x, y \in X$ and $n \in \mathbb{N}$. The first seven terms on the right hand side of (2.6) tend to 1 as $n \rightarrow \infty$ by the definition of $F$ and (N2), and the last term holds

$$
\begin{aligned}
& N^{\prime}\left(D J_{n} f(x, y), \frac{t}{2}\right) \\
\geq & \min \left\{N^{\prime}\left(\frac{D f\left(2^{n} x, 2^{n} y\right)}{2 \cdot 4^{n}}, \frac{t}{8}\right), N^{\prime}\left(\frac{D f\left(-2^{n} x,-2^{n} y\right)}{2 \cdot 4^{n}}, \frac{t}{8}\right),\right. \\
& \left.N^{\prime}\left(\frac{D f\left(2^{n} x, 2^{n} y\right)}{2 \cdot 2^{n}}, \frac{t}{8}\right), N^{\prime}\left(\frac{D f\left(-2^{n} x,-2^{n} y\right)}{2 \cdot 2^{n}}, \frac{t}{8}\right)\right\}
\end{aligned}
$$

for all $x, y \in X$. By (N3) and (2.1), we obtain

$$
\begin{aligned}
N^{\prime}\left(\frac{\left.D f\left( \pm 2^{n} x, \pm 2^{n} y\right)\right)}{2 \cdot 4^{n}}, \frac{t}{8}\right) & =N^{\prime}\left(D f\left( \pm 2^{n} x, \pm 2^{n} y\right), \frac{4^{n} t}{4}\right) \\
& \geq \min \left\{N\left(2^{n} x,\left(\frac{4^{n} t}{8}\right)^{q}\right), N\left(2^{n} y,\left(\frac{4^{n} t}{8}\right)^{q}\right)\right\} \\
& \geq \min \left\{N\left(x, \frac{2^{(2 q-1) n}}{2^{3 q}} t^{q}\right), N\left(y, \frac{2^{(2 q-1) n}}{2^{3 q}} t^{q}\right)\right\}
\end{aligned}
$$

and

$$
N^{\prime}\left(\frac{\left.D f\left( \pm 2^{n} x, \pm 2^{n} y\right)\right)}{2 \cdot 2^{n}}, \frac{t}{8}\right) \geq \min \left\{N\left(x, \frac{2^{(q-1) n}}{2^{3 q}} t^{q}\right), N\left(y, \frac{2^{(q-1) n}}{2^{3 q}} t^{q}\right)\right\}
$$

for all $x, y \in X$ and $n \in \mathbb{N}$. Since $q>1$, together with (N5), we can deduce that the last term of (2.6) also tends to 1 as $n \rightarrow \infty$. It follows from (2.6) that

$$
N^{\prime}(D F(x, y), t)=1
$$

for each $x, y \in X$ and $t>0$. By (N2), this means that $D F(x, y)=0$ for all $x, y \in X$. 
Next we approximate the difference between $f$ and $F$ in a fuzzy sense. For an arbitrary fixed $x \in X$ and $t>0$, choose $0<\varepsilon<1$ and $0<t^{\prime}<t$. Since $F$ is the limit of $\left\{J_{n} f(x)\right\}$, there is $n \in \mathbb{N}$ such that

$$
N^{\prime}\left(F(x)-J_{n} f(x), t-t^{\prime}\right) \geq 1-\varepsilon .
$$

By (2.5), we have

$$
\begin{aligned}
N^{\prime}(F(x)-f(x), t) & \geq \min \left\{N^{\prime}\left(F(x)-J_{n} f(x), t-t^{\prime}\right), N^{\prime}\left(J_{n} f(x)-f(x), t^{\prime}\right)\right\} \\
& \geq \min \left\{1-\varepsilon, N\left(x, \frac{t^{\prime q}}{\left(\frac{1}{2} \sum_{j=0}^{n-1}\left(\frac{2^{p}}{2}\right)^{j}\right)^{q}}\right)\right\} \\
& \geq \min \left\{1-\varepsilon, N\left(x,\left(2-2^{p}\right)^{q} t^{\prime q}\right)\right\} .
\end{aligned}
$$

Because $0<\varepsilon<1$ is arbitrary, we get the inequality (2.2) in this case. Finally, to prove the uniqueness of $F$, let $F^{\prime}: X \rightarrow Y$ be another quadratic-additive mapping satisfying (2.2). Then by (2.3), we get

$$
\left\{\begin{array}{l}
F(x)-J_{n} F(x)=\sum_{j=0}^{n-1}\left(J_{j} F(x)-J_{j+1} F(x)\right)=0 \\
F^{\prime}(x)-J_{n} F^{\prime}(x)=\sum_{j=0}^{n-1}\left(J_{j} F^{\prime}(x)-J_{j+1} F^{\prime}(x)\right)=0
\end{array}\right.
$$

for all $x \in X$ and $n \in \mathbb{N}$. Together with (N4) and (2.2), this implies that

$$
\begin{aligned}
& N^{\prime}\left(F(x)-F^{\prime}(x), t\right) \\
= & N^{\prime}\left(J_{n} F(x)-J_{n} F^{\prime}(x), t\right) \\
\geq & \min \left\{N^{\prime}\left(J_{n} F(x)-J_{n} f(x), \frac{t}{2}\right), N^{\prime}\left(J_{n} f(x)-J_{n} F^{\prime}(x), \frac{t}{2}\right)\right\} \\
\geq & \min \left\{N^{\prime}\left(\frac{(F-f)\left(2^{n} x\right)}{2 \cdot 4^{n}}, \frac{t}{8}\right), N^{\prime}\left(\frac{\left(f-F^{\prime}\right)\left(2^{n} x\right)}{2 \cdot 4^{n}}, \frac{t}{8}\right),\right. \\
& N^{\prime}\left(\frac{(F-f)\left(-2^{n} x\right)}{2 \cdot 4^{n}}, \frac{t}{8}\right), N^{\prime}\left(\frac{\left(f-F^{\prime}\right)\left(-2^{n} x\right)}{2 \cdot 4^{n}}, \frac{t}{8}\right), \\
& N^{\prime}\left(\frac{(F-f)\left(2^{n} x\right)}{2 \cdot 2^{n}}, \frac{t}{8}\right), N^{\prime}\left(\frac{\left(f-F^{\prime}\right)\left(2^{n} x\right)}{2 \cdot 2^{n}}, \frac{t}{8}\right), \\
& \left.N^{\prime}\left(\frac{(F-f)\left(-2^{n} x\right)}{2 \cdot 2^{n}}, \frac{t}{8}\right), N^{\prime}\left(\frac{\left(f-F^{\prime}\right)\left(-2^{n} x\right)}{2 \cdot 2^{n}}, \frac{t}{8}\right)\right\} \\
\geq & \sup _{t^{\prime}<t} N\left(x, 2^{(q-1) n-2 q}\left(2-2^{p}\right)^{q} t^{\prime q}\right)
\end{aligned}
$$

for all $x \in X$ and $n \in \mathbb{N}$. Observe that, for $q=\frac{1}{p}>1$, the last term of the above inequality tends to 1 as $n \rightarrow \infty$ by (N5). This implies that $N^{\prime}\left(F(x)-F^{\prime}(x), t\right)=$ 1 and so we get

$$
F(x)=F^{\prime}(x)
$$

for all $x \in X$ by (N2). 
Case 2. Let $\frac{1}{2}<q<1$ and let $J_{n} f: X \rightarrow Y$ be a mapping defined by

$$
J_{n} f(x)=\frac{1}{2}\left(4^{-n}\left(f\left(2^{n} x\right)+f\left(-2^{n} x\right)\right)+2^{n}\left(f\left(\frac{x}{2^{n}}\right)-f\left(-\frac{x}{2^{n}}\right)\right)\right)
$$

for all $x \in X$. Then we have $J_{0} f(x)=f(x)$ and

$$
\begin{aligned}
J_{j} f(x)-J_{j+1} f(x)= & -\frac{1}{4^{j+2}} D f\left(2^{j} x, 2^{j} x\right)-\frac{1}{4^{j+2}} D f\left(-2^{j} x,-2^{j} x\right) \\
& +2^{j-2} D f\left(\frac{x}{2^{j+1}}, \frac{x}{2^{j+1}}\right)-2^{j-2} D f\left(\frac{-x}{2^{j+1}}, \frac{-x}{2^{j+1}}\right)
\end{aligned}
$$

for all $x \in X$ and $j \geq 0$. If $n+m>m \geq 0$, then we have

$$
\begin{aligned}
& N^{\prime}\left(J_{m} f(x)-J_{n+m} f(x), \sum_{j=m}^{n+m-1}\left(\frac{1}{4}\left(\frac{2^{p}}{4}\right)^{j}+\frac{1}{2^{p}}\left(\frac{2}{2^{p}}\right)^{j}\right) t^{p}\right) \\
\geq & \min \bigcup_{j=m}^{n+m-1}\left\{\operatorname { m i n } \left\{N^{\prime}\left(\frac{D f\left(2^{j} x, 0\right)}{2 \cdot 4^{j+1}}, \frac{2^{j p} t^{p}}{2 \cdot 4^{j+1}}\right),\right.\right. \\
& N^{\prime}\left(\frac{D f\left(-2^{j} x, 0\right)}{2 \cdot 4^{j+1}}, \frac{2^{j p} t^{p}}{2 \cdot 4^{j+1}}\right), N^{\prime}\left(-2^{j-1} D f\left(\frac{x}{2^{j+1}}, 0\right), \frac{2^{j-1} t^{p}}{2^{(j+1) p}}\right), \\
\geq & \min \bigcup_{j=m}^{n+m-1}\left\{N\left(2^{j} x, 2^{j} t\right), N\left(\frac{x}{2^{j+1}}, \frac{t}{2^{j+1}}\right)\right\} \\
= & N(x, t)
\end{aligned}
$$

for all $x \in X$ and $t>0$. In the similar argument following (2.4) of the previous case, we can define the limit $F(x):=N^{\prime}-\lim _{n \rightarrow \infty} J_{n} f(x)$ of the Cauchy sequence $\left\{J_{n} f(x)\right\}$ in the Banach fuzzy space $Y$. Moreover, putting $m=0$ in the above inequality, we have

$$
N^{\prime}\left(f(x)-J_{n} f(x), t\right) \geq N\left(x, \frac{t^{q}}{\left(\sum_{j=0}^{n-1}\left(\frac{1}{4}\left(\frac{2^{p}}{4}\right)^{j}+\frac{1}{2^{p}}\left(\frac{2}{2^{p}}\right)^{j}\right)\right)^{q}}\right)
$$

for each $x \in X$ and $t>0$. To prove that $F$ is a quadratic additive function, we have enough to show that the last term of (2.6) in Case 1 tends to 1 as $n \rightarrow \infty$. By (N3) and (2.1), we get

$$
\begin{aligned}
& N^{\prime}\left(D J_{n} f(x, y), \frac{t}{2}\right) \\
\geq & \min \left\{N^{\prime}\left(\frac{D f\left(2^{n} x, 2^{n} y\right)}{2 \cdot 4^{n}}, \frac{t}{8}\right), N^{\prime}\left(\frac{D f\left(-2^{n} x,-2^{n} y\right.}{2 \cdot 4^{n}}, \frac{t}{8}\right),\right. \\
& \left.N^{\prime}\left(2^{n-1} D f\left(\frac{x}{2^{n}}, \frac{y}{2^{n}}\right), \frac{t}{8}\right), N^{\prime}\left(2^{n-1} D f\left(\frac{-x}{2^{n}}, \frac{-y}{2^{n}}\right), \frac{t}{8}\right)\right\}
\end{aligned}
$$




$$
\begin{array}{r}
\geq \min \left\{N\left(x, 2^{(2 q-1) n-3 q} t^{q}\right), N\left(y, 2^{(2 q-1) n-3 q} t^{q}\right),\right. \\
\left.N\left(x, 2^{(1-q) n-3 q} t^{q}\right), N\left(y, 2^{(1-q) n-3 q} t^{q}\right)\right\}
\end{array}
$$

for each $x, y \in X$ and $t>0$. Observe that all the terms on the right hand side of the above inequality tend to 1 as $n \rightarrow \infty$, since $\frac{1}{2}<q<1$. Hence, together with the similar argument after (2.6), we can say that $D F(x, y)=0$ for all $x, y \in X$. Recall, in Case 1, the inequality (2.2) follows from (2.5). By the same reasoning, we get (2.2) from (2.8) in this case. Now to prove the uniqueness of $F$, let $F^{\prime}$ be another quadratic additive mapping satisfying (2.2). Then, together with (N4), (2.2), and (2.7), we have

$$
\begin{aligned}
& N^{\prime}\left(F(x)-F^{\prime}(x), t\right) \\
&= N^{\prime}\left(J_{n} F(x)-J_{n} F^{\prime}(x), t\right) \\
& \geq \min \left\{N^{\prime}\left(J_{n} F(x)-J_{n} f(x), \frac{t}{2}\right), N^{\prime}\left(J_{n} f(x)-J_{n} F^{\prime}(x), \frac{t}{2}\right)\right\} \\
& \geq \min \{ N^{\prime}\left(\frac{(F-f)\left(2^{n} x\right)}{2 \cdot 4^{n}}, \frac{t}{8}\right),\left(\frac{\left(f-F^{\prime}\right)\left(2^{n} x\right)}{2 \cdot 4^{n}}, \frac{t}{8}\right), \\
& N^{\prime}\left(\frac{(F-f)\left(-2^{n} x\right)}{2 \cdot 4^{n}}, \frac{t}{8}\right), N^{\prime}\left(\frac{\left(f-F^{\prime}\right)\left(-2^{n} x\right)}{2 \cdot 4^{n}}, \frac{t}{8}\right), \\
& N^{\prime}\left(2^{n-1}\left((F-f)\left(\frac{x}{2^{n}}\right)\right), \frac{t}{8}\right), N^{\prime}\left(2^{n-1}\left(\left(f-F^{\prime}\right)\left(\frac{x}{2^{n}}\right)\right), \frac{t}{8}\right), \\
&\left.N^{\prime}\left(2^{n-1}\left((F-f)\left(\frac{-x}{2^{n}}\right)\right), \frac{t}{8}\right), N^{\prime}\left(2^{n-1}\left(\left(f-F^{\prime}\right)\left(\frac{-x}{2^{n}}\right)\right), \frac{t}{8}\right)\right\} \\
& \geq \min \left\{\sup _{t^{\prime}<t} N\left(x, 2^{(2 q-1) n-2 q}\left(\frac{\left(4-2^{p}\right)\left(2^{p}-2\right)}{2}\right)^{q} t^{\prime q}\right),\right. \\
&\left.\sup _{t^{\prime}<t} N\left(x, 2^{(1-q) n-2 q}\left(\frac{\left(4-2^{p}\right)\left(2^{p}-2\right)}{2}\right)^{q} t^{\prime q}\right)\right\}
\end{aligned}
$$

for all $x \in X$ and $n \in \mathbb{N}$. Since $\lim _{n \rightarrow \infty} 2^{(2 q-1) n-2 q}=\lim _{n \rightarrow \infty} 2^{(1-q) n-2 q}=\infty$ in this case, both terms on the right hand side of the above inequality tend to 1 as $n \rightarrow \infty$ by (N5). This implies that $N^{\prime}\left(F(x)-F^{\prime}(x), t\right)=1$ and so $F(x)=F^{\prime}(x)$ for all $x \in X$ by (N2).

Case 3. Finally, we take $0<q<\frac{1}{2}$ and define $J_{n} f: X \rightarrow Y$ by

$$
J_{n} f(x)=\frac{1}{2}\left(4^{n}\left(f\left(2^{-n} x\right)+f\left(-2^{-n} x\right)\right)+2^{n}\left(f\left(\frac{x}{2^{n}}\right)-f\left(-\frac{x}{2^{n}}\right)\right)\right)
$$

for all $x \in X$. Then we have $J_{0} f(x)=f(x)$ and

$$
J_{j} f(x)-J_{j+1} f(x)=\left(4^{j-1}+2^{j-2}\right) D f\left(\frac{x}{2^{j+1}}, \frac{x}{2^{j+1}}\right)
$$




$$
+\left(4^{j-1}-2^{j-2}\right) D f\left(\frac{-x}{2^{j+1}}, \frac{-x}{2^{j+1}}\right)
$$

which implies that if $n+m>m \geq 0$, then

$$
\begin{aligned}
& N^{\prime}\left(J_{m} f(x)-J_{n+m} f(x), \sum_{j=m}^{n+m-1}\left(\frac{4}{2^{p}}\right)^{j} \frac{t^{p}}{2^{p}}\right) \\
\geq & \min \bigcup_{j=m}^{n+m-1}\left\{\operatorname { m i n } \left\{N^{\prime}\left(\left(4^{j-1}+2^{j-2}\right) D f\left(\frac{x}{2^{j+1}}, \frac{x}{2^{j+1}}\right), \frac{\left(2^{2 j-1}+2^{j-1}\right) t^{p}}{2^{(j+1) p}}\right),\right.\right. \\
& \left.\left.N^{\prime}\left(\left(4^{j-1}-2^{j-2}\right) D f\left(-\frac{x}{2^{j+1}},-\frac{x}{2^{j+1}}\right), \frac{\left(2^{2 j-1}-2^{j-1}\right) t^{p}}{2^{(j+1) p}}\right)\right\}\right\} \\
= & \min \bigcup_{j=m}^{n+m-1}\left\{N\left(\frac{x}{2^{j+1}}, \frac{t}{2^{j+1}}\right)\right\}
\end{aligned}
$$

for all $x \in X$ and $t>0$. Similar to the previous cases, it leads us to define the mapping $F: X \rightarrow Y$ by $F(x):=N^{\prime}-\lim _{n \rightarrow \infty} J_{n} f(x)$. Putting $m=0$ in the above inequality, we have

$$
N^{\prime}\left(f(x)-J_{n} f(x), t\right) \geq N\left(x, \frac{t^{q}}{\left(\frac{1}{2^{p}} \sum_{j=0}^{n-1}\left(\frac{4}{2^{p}}\right)^{j}\right)^{q}}\right)
$$

for all $x \in X$ and $t>0$. Notice that

$$
\begin{aligned}
& N^{\prime}(\left.D J_{n} f(x, y), \frac{t}{2}\right) \\
& \geq \min \left\{N^{\prime}\left(\frac{4^{n}}{2} D f\left(\frac{x}{2^{n}}, \frac{y}{2^{n}}\right), \frac{t}{8}\right), N^{\prime}\left(\frac{4^{n}}{2} D f\left(\frac{-x}{2^{n}}, \frac{-y}{2^{n}}\right), \frac{t}{8}\right),\right. \\
&\left.N^{\prime}\left(2^{n-1} D f\left(\frac{x}{2^{n}}, \frac{y}{2^{n}}\right), \frac{t}{8}\right), N^{\prime}\left(2^{n-1} D f\left(\frac{-x}{2^{n}}, \frac{-y}{2^{n}}\right), \frac{t}{8}\right)\right\} \\
& \geq \min \left\{N\left(x, 2^{(1-2 q) n-3 q} t^{q}\right), N\left(y, 2^{(1-2 q) n-3 q} t^{q}\right),\right. \\
&\left.N\left(x, 2^{(1-q) n-3 q} t^{q}\right), N\left(y, 2^{(1-q) n-3 q} t^{q}\right)\right\}
\end{aligned}
$$

for each $x, y \in X$ and $t>0$. Since $0<q<\frac{1}{2}$, all terms on the right hand side tend to 1 as $n \rightarrow \infty$, which implies that the last term of (2.6) tends to 1 as $n \rightarrow \infty$. Therefore, we can say that $D F \equiv 0$. Moreover, using the similar argument after (2.6) in Case 1, we get the inequality (2.2) from (2.9) in this case. To prove the uniqueness of $F$, let $F^{\prime}: X \rightarrow Y$ be another quadratic additive function satisfying (2.2). Then by (2.7), we get

$$
N^{\prime}\left(F(x)-F^{\prime}(x), t\right)
$$




$$
\begin{aligned}
& \geq \min \left\{N^{\prime}\left(J_{n} F(x)-J_{n} f(x), \frac{t}{2}\right), N^{\prime}\left(J_{n} f(x)-J_{n} F^{\prime}(x), \frac{t}{2}\right)\right\} \\
& \geq \min \left\{N^{\prime}\left(\frac{4^{n}}{2}\left((F-f)\left(\frac{x}{2^{n}}\right)\right), \frac{t}{8}\right), N^{\prime}\left(\frac{4^{n}}{2}\left(\left(f-F^{\prime}\right)\left(\frac{x}{2^{n}}\right)\right), \frac{t}{8}\right),\right. \\
& N^{\prime}\left(\frac{4^{n}}{2}\left((F-f)\left(-\frac{x}{2^{n}}\right)\right), \frac{t}{8}\right), N^{\prime}\left(\frac{4^{n}}{2}\left(\left(f-F^{\prime}\right)\left(-\frac{x}{2^{n}}\right)\right), \frac{t}{8}\right), \\
& N^{\prime}\left(2^{n-1}\left((F-f)\left(\frac{x}{2^{n}}\right)\right), \frac{t}{8}\right), N^{\prime}\left(2^{n-1}\left(\left(f-F^{\prime}\right)\left(\frac{x}{2^{n}}\right)\right), \frac{t}{8}\right), \\
&\left.N^{\prime}\left(2^{n-1}\left((F-f)\left(\frac{-x}{2^{n}}\right)\right), \frac{t}{8}\right), N^{\prime}\left(2^{n-1}\left(\left(f-F^{\prime}\right)\left(\frac{-x}{2^{n}}\right)\right), \frac{t}{8}\right)\right\} \\
& \geq \sup _{t^{\prime}<t} N\left(x, 2^{(1-2 q) n-2 q}\left(2^{p}-4\right)^{q} t^{\prime q}\right)
\end{aligned}
$$

for all $x \in X$ and $n \in \mathbb{N}$. Observe that, for $0<q<\frac{1}{2}$, the last term tends to 1 as $n \rightarrow \infty$ by (N5). This implies that $N^{\prime}\left(F(x)-F^{\prime}(x), t\right)=1$ and $F(x)=F^{\prime}(x)$ for all $x \in X$ by (N2).

Remark 2.3. Consider a mapping $f: X \rightarrow Y$ satisfying (2.1) for all $x, y \in X$ and a real number $q<0$. Take any $t>0$. If we choose a real number $s$ with $0<2 s<t$, then we have

$$
N^{\prime}(D f(x, y), t) \geq N^{\prime}(D f(x, y), 2 s) \geq \min \left\{N\left(x, s^{q}\right), N\left(y, s^{q}\right)\right\}
$$

for all $x, y \in X$. Since $q<0$, we have $\lim _{s \rightarrow 0^{+}} s^{q}=\infty$. This implies that

$$
\lim _{s \rightarrow 0^{+}} N\left(x, s^{q}\right)=\lim _{s \rightarrow 0^{+}} N\left(y, s^{q}\right)=1
$$

and so

$$
N^{\prime}(D f(x, y), t)=1
$$

for all $x, y \in X$ and $t>0$. By (N2), it allows us to get $D f(x, y)=0$ for all $x, y \in X$. In other words, $f$ is itself a quadratic additive mapping if $f$ is a fuzzy $q$-almost quadratic-additive mapping for the case $q<0$.

Corollary 2.4. Let $f$ be an even mapping satisfying all of the conditions of Theorem 2.2. Then there is a unique quadratic mapping $F: X \rightarrow Y$ such that

$$
N^{\prime}(F(x)-f(x), t) \geq \sup _{t^{\prime}<t} N\left(x,\left(\left|4-2^{p}\right| t^{\prime}\right)^{q}\right)
$$

for all $x \in X$ and $t>0$, where $p=1 / q$.

Proof. Let $J_{n} f$ be defined as in Theorem 2.2. Since $f$ is an even mapping, we obtain

$$
J_{n} f(x)= \begin{cases}\frac{f\left(2^{n} x\right)+f\left(-2^{n} x\right)}{2 \cdot 4^{n}} & \text { if } 0<q<\frac{1}{2} \\ \frac{1}{2}\left(4^{n}\left(f\left(2^{-n} x\right)+f\left(-2^{-n} x\right)\right)\right) & \text { if } q>\frac{1}{2}\end{cases}
$$


for all $x \in X$. Notice that $J_{0} f(x)=f(x)$ and

$J_{j} f(x)-J_{j+1} f(x)= \begin{cases}\frac{-1}{4^{j+2}}\left(D f\left(2^{j} x, 2^{j} x\right)+D f\left(-2^{j} x,-2^{j} x\right)\right) & \text { if } 0<q<\frac{1}{2}, \\ 4^{j-1}\left(D f\left(\frac{x}{2^{j+1}}, \frac{x}{2^{j+1}}\right)+D f\left(\frac{-x}{2^{j+1}}, \frac{-x}{2^{j+1}}\right)\right) & \text { if } q>\frac{1}{2}\end{cases}$

for all $x \in X$ and $j \in \mathbb{N} \cup\{0\}$. From these, using the similar method in Theorem 2.2 , we obtain the quadratic-additive mapping $F$ satisfying (2.10). Notice that $F$ is also even, $F(x):=N^{\prime}-\lim _{n \rightarrow \infty} J_{n} f(x)$ for all $x \in X$, and $D F(x, y)=0$ for all $x, y \in X$. Hence, we get

$$
F(x+y)+F(x-y)-2 F(x)-2 F(y)=\frac{1}{2} D F(x, y)=0
$$

for all $x, y \in X$. This means that $F$ is a quadratic mapping.

Corollary 2.5. Let $f$ be an odd mapping satisfying all of the conditions of Theorem 2.2. Then there is a unique additive mapping $F: X \rightarrow Y$ such that

$$
N^{\prime}(F(x)-f(x), t) \geq \sup _{t^{\prime}<t} N\left(x,\left(\left|2-2^{p}\right| t^{\prime}\right)^{q}\right)
$$

for all $x \in X$ and $t>0$, where $p=1 / q$.

Proof. Let $J_{n} f$ be defined as in Theorem 2.2. Since $f$ is an odd mapping, we obtain

$$
J_{n} f(x)= \begin{cases}\frac{f\left(2^{n} x\right)+f\left(-2^{n} x\right)}{2^{n+1}} & \text { if } 0<q<1 \\ 2^{n-1}\left(f\left(2^{-n} x\right)+f\left(-2^{-n} x\right)\right) & \text { if } q>1\end{cases}
$$

for all $x \in X$. Notice that $J_{0} f(x)=f(x)$ and

$J_{j} f(x)-J_{j+1} f(x)= \begin{cases}\frac{1}{2^{j+3}}\left(D f\left(-2^{j} x,-2^{j} x\right)-D f\left(2^{j} x, 2^{j} x\right)\right) & \text { if } 0<q<1 \\ 2^{j-2}\left(D f\left(\frac{x}{2^{j+1}}, \frac{x}{2^{j+1}}\right)-D f\left(\frac{-x}{2^{j+1}}, \frac{-x}{2^{j+1}}\right)\right) & \text { if } q>1\end{cases}$

for all $x \in X$ and $j \in \mathbb{N} \cup\{0\}$. From these, using the similar method in Theorem 2.2 , we obtain the quadratic-additive mapping $F$ satisfying (2.11). Notice that $F$ is also odd, $F(x):=N^{\prime}-\lim _{n \rightarrow \infty} J_{n} f(x)$ for all $x \in X$, and $D F(x, y)=0$ for all $x, y \in X$. Hence, we get

$$
F(x+y)-F(x)-F(y)=\frac{1}{2} D F(x, y)=0
$$

for all $x, y \in X$. This means that $F$ is an additive mapping.

We can use Theorem 2.2 to get a classical result in the framework of normed spaces. Let $(X,\|\cdot\|)$ be a normed linear space. Then we can define a fuzzy norm $N_{X}$ on $X$ by following

$$
N_{X}(x, t)= \begin{cases}0, & t \leq\|x\| \\ 1, & t>\|x\|\end{cases}
$$

where $x \in X$ and $t \in \mathbb{R}$, see [17]. Suppose that $f: X \rightarrow Y$ is a mapping into a Banach space $(Y,\||\cdot|\|)$ such that

$$
\||| D f(x, y)\|\|\leq\| x\left\|^{p}+\right\| y \|^{p}
$$


for all $x, y \in X$, where $p>0$ and $p \neq 1,2$. Let $N_{Y}$ be a fuzzy norm on $Y$. Then we get

$$
N_{Y}(D f(x, y), s+t)= \begin{cases}0, & s+t \leq\|\mid\| D f(x, y)\|\| \\ 1, & s+t>\||| D f(x, y)\| \|\end{cases}
$$

for all $x, y \in X$ and $s, t \in \mathbb{R}$. Consider the case $N_{Y}(D f(x, y), s+t)=0$. This implies that

$$
\|x\|^{p}+\|y\|^{p} \geq\|\mid D f(x, y)\| \| \geq s+t
$$

and so either $\|x\|^{p} \geq s$ or $\|y\|^{p} \geq t$ in this case. Hence, for $q=\frac{1}{p}$, we have

$$
\min \left\{N_{X}\left(x, s^{q}\right), N_{X}\left(y, t^{q}\right)\right\}=0
$$

for all $x, y \in X$ and $s, t>0$. Therefore, in every case, the inequality

$$
N_{Y}(D f(x, y), s+t) \geq \min \left\{N_{X}\left(x, s^{q}\right), N_{X}\left(y, t^{q}\right)\right\}
$$

holds. It means that $f$ is a fuzzy $q$-almost quadratic additive mapping, and by Theorem 2.2 , we get the following stability result.

Corollary 2.6. Let $(X,\|\cdot\|)$ be a normed linear space and let $(Y,\|\| \cdot \| \mid)$ be a Banach space. If

$$
\left\|\left|D f(x, y)\|\mid \leq\| x\left\|^{p}+\right\| y \|^{p}\right.\right.
$$

for all $x, y \in X$, where $p>0$ and $p \neq 1,2$, then there is a unique quadraticadditive mapping $F: X \rightarrow Y$ such that

$$
\|F(x)-f(x) \mid\| \leq \begin{cases}\frac{\|x\|^{p}}{2-2^{p}} & \text { if } 0<p<1, \\ \frac{2\|x\|^{p}}{\left(2-2^{p}\right)\left(2^{p}-4\right)} & \text { if } 1<p<2, \\ \frac{\|x\| \|^{p}}{2^{p}-4} & \text { if } 2<p\end{cases}
$$

for all $x \in X$.

\section{References}

[1] T. Aoki, On the stability of the linear transformation in Banach spaces, J. Math. Soc. Japan 2 (1950), 64-66.

[2] T. Bag and S. K. Samanta, Finite dimensional fuzzy normed linear spaces, J. Fuzzy Math. 11 (2003), no. 3, 687-705.

[3] S. C. Cheng and J. N. Mordeson, Fuzzy linear operator and fuzzy normed linear spaces, Bull. Calcutta Math. Soc. 86 (1994), no. 5, 429-436.

[4] S. Czerwik, On the stability of the quadratic mapping in normed spaces, Abh. Math. Sem. Univ. Hamburg 62 (1992), 59-64.

[5] P. Găvruta, A generalization of the Hyers-Ulam-Rassias stability of approximately additive mappings, J. Math. Anal. Appl. 184 (1994), no. 3, 431-436.

[6] D. H. Hyers, On the stability of the linear functional equation, Proc. Natl. Acad. Sci. USA 27 (1941), 222-224.

[7] K.-W. Jun and Y.-H. Lee, A generalization of the Hyers-Ulam-Rassias stability of the Pexiderized quadratic equations. II, Kyungpook Math. J. 47 (2007), no. 1, 91-103.

[8] A. K. Katsaras, Fuzzy topological vector spaces II, Fuzzy Sets and Systems 12 (1984), no. 2, 143-154.

[9] G.-H. Kim, On the stability of functional equations with square-symmetric operation, Math. Inequal. Appl. 4 (2001), no. 2, 257-266. 
[10] H.-M. Kim, On the stability problem for a mixed type of quartic and quadratic functional equation, J. Math. Anal. Appl. 324 (2006), no. 1, 358-372.

[11] I. Kramosil and J. Michalek, Fuzzy metric and statistical metric spaces, Kybernetika (Prague) 11 (1975), no. 5, 326-334.

[12] Y.-H. Lee, On the Hyers-Ulam-Rassias stability of the generalized polynomial function of degree 2, J. Chuncheong Math. Soc. 22, (2009) 201-209.

[13] - On the stability of the monomial functional equation, Bull. Korean Math. Soc. 45 (2008), no. 2, 397-403

[14] Y. H. Lee and K. W. Jun, A generalization of the Hyers-Ulam-Rassias stability of Jensen's equation, J. Math. Anal. Appl. 238 (1999), no. 1, 305-315.

[15] _ A generalization of the Hyers-Ulam-Rassias stability of the Pexider equation, J. Math. Anal. Appl. 246 (2000), no. 2, 627-638

[16] - On the stability of approximately additive mappings, Proc. Amer. Math. Soc. 128 (2000), no. 5, 1361-1369.

[17] A. K. Mirmostafaee and M. S. Moslehian, Fuzzy almost quadratic functions, Results Math. 52 (2008), no. 1-2, 161-177.

[18] _ Fuzzy versions of Hyers-Ulam-Rassias theorem, Fuzzy Sets and Systems 159 (2008), no. 6, 720-729

[19] C.-G. Park, On the stability of the Cauchy additive and quadratic type functional equation, to appear.

[20] Th. M. Rassias, On the stability of the linear mapping in Banach spaces, Proc. Amer. Math. Soc. 72 (1978), no. 2, 297-300.

[21] F. Skof, Local properties and approximations of operators, Rend. Sem. Mat. Fis. Milano 53 (1983), 113-129.

[22] S. M. Ulam, A Collection of Mathematical Problems, Interscience, New York, 1968.

SunSoOK Jin

Department of Mathematics Education

Gonguu National University of Education

GongJu 314-711, Korea

E-mail address: ssjin@gjue.ac.kr

YANG-Hi LEE

Department of Mathematics Education

GonguU National University of Education

GonguU 314-711, KoreA

E-mail address: lyhmzi@gjue.ac.kr 